\title{
FUNCIONES, RESPONSABILIDADES, PROPUESTAS Y EXPECTATIVAS DE LA LABOR TUTORIAL: OPINIONES DE LOS DOCENTES Y ESTUDIANTES DE LA ESCUELA DE EDUCACIÓN FÍSICA DE LA PONTIFICIA UNIVERSIDAD CATÓLICA DE VALPARAISO
}

\section{DUTIES, RESPONSIBILITIES AND EXPECTATIONS OF THE PROPOSED WORK TUTORIAL: OPINIONS OF TEACHERS AND STUDENTS OF THE SCHOOL OF PHYSICAL EDUCATION OF PONTIFICIA UNIVERSIDAD CATÓLICA DE VALPARAISO}

\author{
Poblete Gálvez, Carolina \& Gamboa Jiménez, Rodrigo \\ Laboratorio de Aprendizaje y Desarrollo Motor. Escuela de Educación Física. Pontificia Universidad Católica de Valparaíso.
}

POBLETE G.C. \& GAMBOA J.R. Funciones, responsabilidades, propuestas y expectativas de la labor tutorial: opiniones de los docentes y estudiantes de la Escuela de Educación Física de la Pontificia Universidad Católica de Valparaíso. Mot.Hum. 13(1): 13-19; 2012.

\begin{abstract}
RESUMEN
La presente investigación indaga sobre la opinión de estudiantes y docentes de la Escuela de Educación Física de la Pontificia Universidad Católica de Valparaíso, respecto a funciones y responsabilidades de los actores que participan de los procesos al interior de un plan tutorial; además de las expectativas y sugerencias que poseen de dichos procesos. La muestra esta conformada por 200 estudiantes de la Unidad Académica y 9 docentes que cumplen o han cumplido la labor de tutor. Para la recolección de la información se ha utilizado un cuestionario de opinión compuesto por 2 preguntas cerradas y 4 abiertas. Para el procesamiento de la información, se ha realizado un análisis cuantitativo de las preguntas cerradas, distribución de frecuencia simple expresada en porcentajes, y un análisis cualitativo de las preguntas abiertas, confección de un sistema de categorías por medio del software Nvivo en su versión 9.0. En relación a los resultados, fundamentalmente se centran en que la tutoría debe ser un proceso de acompañamiento sistemático, no sólo en temas administrativo y académicos, si no que debe transformarse en un trabajo entre docente y estudiantes que colabore con el desarrollo académico, profesional y personal de quienes son parte del proceso, en el cual los actores deben asumir proactivamente funciones y responsabilidades que le son propia a la labor tutorial.
\end{abstract}

Palabras Clave: Tutorías, plan tutorial, proceso formativo, tutor, función Tutorial.

\begin{abstract}
The present investigation investigates on the opinion of students and teachers of the School of Physical Education of the Pontificia Universidad Catolica de Valparaiso, with regard to functions and responsibilities of the actors who take part from the processes to the interior of a plan tutorial; besides the expectations and suggestions that they possess of the above mentioned processes. The this sample shaped by 200 students of the Academic Unit and 9 teachers who expire or have fulfilled the tutor's labor. For the compilation of the information there has been in use a questionnaire of opinion composed by 2 closed questions and 4 opened ones. For the processing of the information, there has been realized a quantitative analysis of the closed questions, distribution of simple frequency expressed in percentages, and a qualitative analysis of the opened questions, confection of a system of categories by means of the software Nvivo in his version 9.0. In relation to the results, fundamentally they centre that the tutorship must be a process of systematic accompaniment, not only in topics administrative officer and academicians, if not that must transform in a work between teacher and students that he collaborates with the academic, professional and personal development of those who are a part of the process, which the actors must assume proactively functions and responsibilities that him are own to the labor tutorial.
\end{abstract}

Key Words: Tutorships, plan tutorial, formative process, tutor, function tutorial. 
POBLETE G.C. \& GAMBOA J.R. Funciones, responsabilidades, propuestas y expectativas de la labor tutorial: opiniones de los docentes y estudiantes de la Escuela de Educación Física de la Pontificia Universidad Católica de Valparaíso. Mot.Hum. 13(1): 13-19; 2012.

\section{INTRODUCCIÓN}

Tutorías o plan tutorial son un neologismo, entendido como palabras que en el contexto educativo actual, se le ha asignado una nueva labor, más allá de lo administrativo, un acompañamiento en lo académico, profesional y personal que beneficiará a todos los actores involucrados.

En el contexto de la educación superior, la tutoría es un proceso de acompañamiento personalizado a los estudiantes durante su formación profesional, mediado por un docente quien facilita el desarrollo en los ámbitos mencionados de dichos estudiantes, $(1,2,3,4)$.

En este proceso de acompañamiento, se produce un encuentro y simbiosis entre dos o más personas, en el que por medio del reconocimiento mutuo, se posibilitan diversas experiencias de desarrollo de aptitudes, de lo afectivo, de conocimientos, valores sociales, entre otras, (5). En tanto tal, es un proceso que está vinculado con la relación persona a persona, y que repercute en la vida social de los actores partícipes de la experiencia, requiriendo responsabilidad y compromiso de ellos. Es un proceso humanizante y altamente solidario donde la empatía juega un rol crucial.

La tutoría como función docente, trasciende los conceptos de asesoría (6), y orientación (7). En este sentido, la Universidad Veracruciana, conceptualiza la asesoría como la relación establecida en forma eventual y no sistematizada, y la orientación como un sistema de guía, que depende de un modelo educativo adoptado y que alimenta la tutoría, (8).

Se puede señalar entonces que el término tutoría trasciende de estos conceptos, dado que es un plan estructurado entre tutor y tutorado, y acorde con los lineamientos de un programa determinado que cumple la tarea de orientar, asesorar y acompañar sistemáticamente al estudiante, con las funciones y responsabilidades que ello implica, (6).

La función tutorial es entendida como las acciones de acompañamiento durante el proceso formativo que implica el cumplimiento de las tareas, es decir, el logro de los mecanismos necesarios para el desarrollo integral de los estudiantes, $(5,9)$. Por ejemplo, el tutor debe propiciar y facilitar la formación en valores, de actitudes y hábitos positivos, de habilidades intelectuales, además de promover mayores instancias de diálogo que generen más instancias de reflexión. Dichas acciones posiblemente contribuirán a minimizar la deserción y rezago de los estudiantes, y así, aumentar los índices de titulación. Si bien, se pueden señalar varias funciones más a la la- bor tutorial tal y como lo plantean otros autores $(4,10$, $11,12,13,14)$, lo central radica en que el tutor como profesional de apoyo tenga como función, facilitar el desarrollo personal, académico y social de los estudiantes, acompañarlos en la realización del trayecto de sus estudios, y colaborar a desarrollar su identidad profesional.

La tutoría busca principalmente evitar el fracaso del estudiante en su desempeño en la educación, propiciando contextos y climas adecuados que acojan las necesidades antes de que impidan el poder ser abordadas, basandose en el reconocimiento de las diferencias individuales, situación que reclama una atención personalizada, aunque no individualista, con sentido humanista y de cara a la proyección futura del estudiante, (15). En este contexto, el estudiante debe ser un actor proactivo y responsable en las tareas y roles que se le asigne o se concuerden conjuntamente con sus pares y/o tutor.

Ante estas funciones y responsabilidades que implica la tutoría tal y como se concibe hoy en día, surgen interrogantes tales como ¿Qué implica este acompañamiento? ¿Qué significa para una institución acompañar sistemáticamente a sus estudiantes?, ¿Qué implica para los docentes y estudiantes vivenciar estos procesos?.

Preguntas que merecen entender también el contexto social y cultural, sus implicancias, y su rol al interior de la educación en Chile. Educación que ha evolucionado desde la cobertura, a la calidad, entendiendo calidad en términos de la apertura a competencias necesarias para que cada estudiante pueda insertarse de manera adecuada en los ámbitos de desempeño profesional, (16).

La idea de tutoría que nace y se basa en el reconocimiento de la persona y con un sentido humanista (15), es motivo de análisis también en las esferas mundiales, como un complemento necesario para los procesos de enseñanza y de aprendizaje, donde se ha desarrollado esta temática como complemento importante de la calidad de la educación. Por ejemplo, en la Universidad Complutense de Madrid, a partir de sus investigaciones plantean la importancia de conocer y entender este proceso como parte importante de la formación profesional, (10). Y en Cuba, ésta labor ha transitado en su sentido desde la asesoría científica metodológica, hacia adquirir un valor en el ámbito de la formación de la cultura general e integral de los estudiantes, (17).

En este contexto de entender las tutorías como parte importante del proceso de formación profesional, nutrido del contexto político, social y cultural de la sociedad en que se inserta la entidad educativa que lo implementa, en el presente estudio se indaga en una realidad particular 
POBLETE G.C. \& GAMBOA J.R. Funciones, responsabilidades, propuestas y expectativas de la labor tutorial: opiniones de los docentes y estudiantes de la Escuela de Educación Física de la Pontificia Universidad Católica de Valparaíso. Mot.Hum. 13(1): 13-19; 2012.

las funciones y responsabilidades de los actores involucrados en estos procesos, y su grado de acuerdo con cada una de ellas. Además de establecer cuales son la principales expectativas y sugerencias que poseen estudiantes $\mathrm{y}$ docentes tutores.

\section{MATERIAL Y MÉTODOS}

El presente estudio de carácter cuantitativo, en el cual se utilizan recursos cualitativos, específicamente a través de un levantamiento de categorías de análisis, con el propósito de enriquecer los hallazgos de investigación.

La muestra es de carácter no probabilística intencionada y esta conformada por 200 estudiantes y 9 docentes de la Escuela de Educación Física de la Pontificia Universidad Católica de Valparaíso. Compuesta por 65 estudiantes de primer año, 44 de segundo, 46 de tercero, 34 de cuarto y 11 de quinto, que representa el $61,91 \%$ del total de la población; además de 9 decentes que han cumplido la labor de tutor.

En el proceso de recolección de la información, se aplicó un cuestionario de opinión compuesto por 2 preguntas de carácter cerrado, y 4 de tipo abiertas. Indaga sobre la opinión que poseen estudiantes y docentes respecto a las funciones del tutor, responsabilidad de los estudiantes, y expectativas y sugerencias de mejora de la labor tutorial. Dicho cuestionario fue validado por expertos en la temática y aplicado en pruebas piloto.

Para el análisis de la información, se ha realizado un análisis cuantitativo por medio de una distribución de frecuencia simple expresada en porcentajes de la información que emana de la sección de preguntas cerradas, de modo de determinar el grado de acuerdo de los consultados.

Y un análisis cualitativo por medio del levantamiento de un sistema de categorías que emergen de la investigación a través el software Nvivo 9.0, y que emana de la sección de preguntas abiertas.

\section{RESULTADOS}

El primer análisis cuantitativo de la información, es en relación a las opiniones de docentes y estudiantes respecto a los porcentajes de aprobación de las funciones que le corresponde a un tutor en la Escuela de Educación Física de la Pontificia Universidad Católica de Valparaíso, tabla ${ }^{\circ}{ }^{\circ}$.

1.1. Acompañar a sus estudiantes en el proceso formativo.

$100,00 \% \quad 82,38 \%$

1.2. Proponer planes de trabajo tendientes a mejorar el desempeño de todos los estudiantes y tutores.

1.3. Publicar claramente su horario de atención semanal.

1.4. Acompañar y guiar al estudiante durante los períodos de inscripción y modificación de asignaturas.

$100,00 \%$

.5. Orientar al estudiante en la elección del currículo de acuerdo a sus necesidades y aptitudes.

1.6. Supervisar el currículo del estudiante, teniendo presente el tiempo máximo para cumplir los créditos demandados.

1.7. Resolver las consultas del estudiante acerca de los problemas curriculares presentados.

1.8. Solicitar reuniones para determinar si procede la otorgación de nuevas oportunidades de cursar asignaturas o eximiciones de un pre-requisito o sanción académica.

1.9. Evaluar sistemáticamente la labor tutorial para retroalimentar el proceso.

$77,78 \% \quad 89,25 \%$

$77,78 \% \quad 60,71 \%$

$66,67 \% \quad 54,01 \%$

$88,89 \% \quad 98,88 \%$

$55,56 \% \quad 56,27 \%$

$44,44 \% \quad 50,26 \%$

Tabla I: Porcentaje de acuerdo en relación a las funciones del tutor.

Se puede observar que en los ítemes en los que estudiantes y docentes poseen mayores porcentajes de acuerdo, son en las funciones relacionadas en lo académico de acompañar a los estudiantes en su proceso formativo, orientarlo en la elección del currículo, y resolver consultas sobre este último. Y desde lo administrativo, fundamentalmente que den a conocer sus horarios de atención.
Aquellas funciones que poseen los menores porcentajes de acuerdo, son fundamentalmente las de gestionar reuniones, supervisar el avance curricular del estudiantes y retroalimentar el proceso tutorial en beneficio del mismo. En la tabla II, se realiza una especificación de los porcentajes según generación, en cuanto al grado de acuerdo en las funciones de un tutor, con el propósito de obtener un panorama global de las concordancias y disonancias. 
POBLETE G.C. \& GAMBOA J.R. Funciones, responsabilidades, propuestas y expectativas de la labor tutorial: opiniones de los docentes y estudiantes de la Escuela de Educación Física de la Pontificia Universidad Católica de Valparaíso. Mot.Hum. 13(1): 13-19; 2012.

\begin{tabular}{|c|c|c|c|c|c|c|c|c|c|}
\hline AÑO & 1,1 & 1,2 & 1,3 & 1,4 & 1,5 & 1,6 & 1,7 & 1,8 & 1,9 \\
\hline $1^{o}$ & $90,77 \%$ & $49,23 \%$ & $92,31 \%$ & $78,46 \%$ & $64,62 \%$ & $53,85 \%$ & $93,85 \%$ & $69,23 \%$ & $60,00 \%$ \\
\hline $2^{\circ}$ & $84,09 \%$ & $34,09 \%$ & $93,18 \%$ & $95,45 \%$ & $43,18 \%$ & $50,00 \%$ & $95,45 \%$ & $50,00 \%$ & $47,73 \%$ \\
\hline $3^{\circ}$ & $91,30 \%$ & $39,13 \%$ & $97,83 \%$ & $93,48 \%$ & $67,39 \%$ & $58,70 \%$ & $100,00 \%$ & $54,35 \%$ & $47,83 \%$ \\
\hline $4^{\circ}$ & $91,18 \%$ & $38,24 \%$ & $94,12 \%$ & $97,06 \%$ & $64,71 \%$ & $52,94 \%$ & $94,12 \%$ & $44,12 \%$ & $41,18 \%$ \\
\hline $5^{\circ}$ & $54,55 \%$ & $45,45 \%$ & $90,91 \%$ & $81,82 \%$ & $63,64 \%$ & $54,55 \%$ & $100,00 \%$ & $63,64 \%$ & $54,55 \%$ \\
\hline
\end{tabular}

Es interesante observar, que si bien existe una diferencia del porcentaje entre las distintas generaciones frente a cada una de las funciones a cumplir por el tutor, la tendencia de aprobación es similar, a excepción de la función de acompañar el proceso formativo de los estudiantes, en la cual el quinto año de la carrera le asigna una aprobación menor entre 30 y 40 puntos porcentuales aproximadamente; y en la función orientar a los estudi- antes en la elección del currículo, en la que segundo año le otorgan una aprobación menor de alrededor de 20 a 25 puntos porcentuales aproximadamente.

En relación a las responsabilidades de los estudiantes en sus procesos tutoriales, en la tabla III se señala el porcentaje de acuerdo en relación a dichas responsabilidades.

2.1. Informarse de los procedimientos académico, curriculares y administrativos.

2.2. Mantener una comunicación permanente con su tutor, respetando las formalidades

y los tiempos de atención semanal, para solicitar orientación que como estudiante podría requerir.

2.3. Manifestar disposición y cumplimiento con las tareas que se designen para el resguardo de su

2.4. Formalizar inscripción de asignaturas solo con su tutor designado.

2.5. Canalizar los actos de reclamo o desacuerdo de la función tutorial por medio de carta formal $88,89 \% \quad 65,04 \%$ a la jefatura de docencia con copia a su respectivo tutor.

2.6. Evaluar sistemáticamente la labor tutorial para retroalimentar el procesos.

Tabla III: Porcentaje de acuerdo en relación a las responsabilidades del estudiante

Es interesante observar, que tanto en docente como estudiantes, el porcentaje de aprobación respecto a las responsabilidades del estudiantado en sus procesos tutoriales es alto, independiente que dicha responsabilidad sea académica o administrativa.
En la tabla IV, se discriminan los resultados en relación a las responsabilidades de los estudiantes en sus procesos tutoriales, en porcentajes de acuerdos según generación, con la finalidad de obtener una visión global de las concordancias y disonancias.

\begin{tabular}{|lcccccc|}
\hline AÑO & 1,1 & 1,2 & 1,3 & 1,4 & 1,5 & 1,6 \\
$\mathbf{1}^{\mathbf{0}}$ & $90,77 \%$ & $49,23 \%$ & $92,31 \%$ & $78,46 \%$ & $64,62 \%$ & $53,85 \%$ \\
$\mathbf{2}^{\mathbf{o}}$ & $84,09 \%$ & $34,09 \%$ & $93,18 \%$ & $95,45 \%$ & $43,18 \%$ & $50,00 \%$ \\
$\mathbf{3}^{\mathbf{0}}$ & $91,30 \%$ & $39,13 \%$ & $97,83 \%$ & $93,48 \%$ & $67,39 \%$ & $58,70 \%$ \\
$\mathbf{4}^{\mathbf{0}}$ & $91,18 \%$ & $38,24 \%$ & $94,12 \%$ & $97,06 \%$ & $64,71 \%$ & $52,94 \%$ \\
$\mathbf{5}^{\mathbf{0}}$ & $54,55 \%$ & $45,45 \%$ & $90,91 \%$ & $81,82 \%$ & $63,64 \%$ & $54,55 \%$ \\
\hline
\end{tabular}

Tabla IV: Porcentaje de acuerdo en relación a las responsabilidades del estudiante por generación. 
POBLETE G.C. \& GAMBOA J.R. Funciones, responsabilidades, propuestas y expectativas de la labor tutorial: opiniones de los docentes y estudiantes de la Escuela de Educación Física de la Pontificia Universidad Católica de Valparaíso. Mot.Hum. 13(1): 13-19; 2012.

Se puede observar, que se presenta la misma tendencia que en la gráfica ${ }^{\circ}$ I. Si bien existen diferencias porcentuales en el grado de aprobación frente a las distintas responsabilidades del estudiante en sus procesos tutoriales, la tendencia de aprobación en cada generación es similar. Las principales diferencias se presentan en las responsabilidades de cumplir con las tareas tutoriales, en la cual segundo y cuarto año le entregan una aprobación menor de alrededor de 17 a 24 puntos porcentuales aproximadamente; en los procesos de inscripción de asignaturas, en la que primero y quinto año le asignan una aprobación menor de alrededor de 16 puntos porcentuales aproximadamente; y en canalizar las discrepancias de los estudiantes a través de los canales formales de la Unidad Académica, en la cual tercero y quinto año le dan un grado de aprobación mayor de alrededor de 14 a 18 puntos porcentuales aproximadamente. Del análisis cualitativo de las preguntas abiertas realizadas a estudiantes y docentes, se ha levantado un sistema de categorías y subcategorias que se señala en la tabla $\mathrm{V}$.
CATEGORIAS

1. Funciones del tutor.

2. Responsabilidades del estudiante.

3. Propuesta de roles y funciones.

4. Expectativas del plan tutorial

\section{SUBCATEGORIAS}

1.1. Acompañamiento académico.

1.2. Acompañamiento administrativo y personal.

2.1. Académicas.

2.2. Actitudinales.

3.1. Acompañamiento y representatividad.

3.2. Relación tutor estudiante.

4.1. Mejora en temas académicos y administrativos.

4.2. Relación entre tutores.

4.3. Relaciones tutor estudiante.

Tabla V: Categorías y subcategorías que emergen del análisis de las respuestas de estudiantes y docentes.

Dentro de las funciones del tutor, en relación al acompañamiento académico, los docentes relevan fundamentalmente, que los tutores puedan compartir con quienes imparten docencia en su generación, las características que distinguen a dicho grupo, de modo que puedan contextualizar su quehacer profesional, y realizar reuniones a lo menos una vez en cada periodo académico con su generación, para conocer de manera general el estado de avance y posibles problemáticas del grupo. Por su parte, los estudiantes relevan dos grandes ideas: formular proyectos de interés tendientes a beneficiar los procesos formativos, y mediar en problemáticas de asignatura entre sus tutorandos y docentes.

En el acompañamiento administrativo y personal, los docentes fundamentalmente señalan que deben fomentar el trabajo en grupo y colaborativo entre estudiantes. Y el estudiantado releva que el tutor debe colaborar en los trámites administrativos, acompañar y participar con sus estudiantes en actividades no académicas, como viajes de estudio, semana de escuela, entre otros.

En la categoría de responsabilidades académicas del estudiante, los docentes plantean como principales responsabilidades que el estudiantado debe informar a su tutor sobre toda acción que afecte su situación al interior de la Unidad Académica, y propongan estrategias a su tutor con el propósito de desarrollarse en áreas de interés en beneficio directo de sus procesos formativos .

De las responsabilidades actitudinales de los estudiantes, ellos señalan tres ideas centrales, mantener respeto hacia su tutor, disponerse positivamente al trabajo colaborativo, y ser criteriosos para solicitar ayuda de su tutor, recurriendo a él sólo en caso de verdadera importancia.

Para la categoría de propuestas de roles y funciones, específicamente en lo relacionado con el acompañamiento y representatividad del tutor, los docentes proponen cuatro ideas centrales: existencia de un tutor para los estudiantes deportistas destacados, el tutor establezca comunicación con los servicios de la institución con el objetivo de informarse sobre otras posibilidades de apoyo al estudiante, la labor tutorial esté dentro de un marco de mayor intervención con el fin de mejorar la calidad de la formación, y que se realicen a lo menos dos reuniones de tutoría al año.

Por su parte, los estudiantes relevan de manera especial y proponen que el tutor organice reuniones periódicas, envíe información de manera individual o con los delegados de generación, informar temas de interés como cursos de perfeccionamiento, seminarios, programas de intercambio, entre otros. 
POBLETE G.C. \& GAMBOA J.R. Funciones, responsabilidades, propuestas y expectativas de la labor tutorial: opiniones de los docentes y estudiantes de la Escuela de Educación Física de la Pontificia Universidad Católica de Valparaíso. Mot.Hum. 13(1): 13-19; 2012.

Respecto a la relación tutor estudiante, los estudiantes hacen hincapié en la idea que el tutor apoye activamente a su generación, y organice periódicamente actividades en temas académicos y extra-académicos.

Finalmente, para la categoría de expectativas sobre la labor tutorial, en lo relacionado con la mejora en temas académicos y administrativos, los docentes mencionan cuatro ideas: que los estudiantes puedan optimizar su tiempo de estancia en la carrera, orientarlos a proseguir estudios y/o perfeccionarse, ser un apoyo a los estudiantes en sus procesos formativos, y que oriente a otros tutores que asumirán mas adelante esta función académica.

Por su parte, el estudiantado señala y releva más expectativas que los docentes tutores, entre las cuales destacan: que el tutor ayude, guíe y acompañe a los estudiantes en temas académicos, administrativos y personales; mantengan informado a los estudiantes sobre temas de interés de la carrera; tenga un mayor compromiso con su labor tutorial; que participe en acciones organizadas por su generación; represente y sea el portavoz de las problemáticas de su generación ante el consejo y dirección de escuela; y defina un horario establecido de atención a sus estudiantes.

De la relación entre tutores, los docentes relevan que esperan que la labor tutorial sea una instancia para mejorar las relaciones entre tutores, e incluso entre los miembros de la Unidad Académica. Pero los estudiantes si señalan expectativas respecto a las relaciones entre tutor y estudiantes, en las que mencionan que esperan que la labor tutorial colabore en lograr mayor cercanía con sus tutores, de manera que les brinden confianza en sus procesos de formación profesional a través de un apoyo mutuo.

\section{DISCUSIÓN}

A la labor tutorial, como tarea humanizante y altamente solidaria en la cual se produce una relación persona a persona, le subyacen tareas y responsabilidades que le son propias, y deben ser asumidas proactivamente por quienes participan, docentes y estudiantes, de modo de favorecer el desarrollo académico, profesional y personal de dichos participantes.

En este contexto, tal como lo señalan algunos autores $(9,5,4,11,13)$, los sujetos participantes del presente estudio también coinciden en una serie de tareas que le son propias fundamentalmente al tutor, dentro de las cuales se relevan: acompañar a los estudiantes en su proceso formativo, orientarlo en la elección del currículo y aclarar interrogantes sobre este, compartir con otros docentes las características que distinguen a su grupo para que puedan contextualizar su quehacer en el aula, diseñar proyectos de interés que beneficiar los procesos formativos, fomentar el trabajo en grupo y colaborativo, con el propósito de colaborar con el desarrollo integral de sus estudiantes, $(1,2,3,4,5,9)$.

Conjuntamente a las funciones atribuidas al tutor, el estudiante debe asumir un rol proactivo, de modo de cumplir responsablemente en las tareas que le son asignadas. De este modo, los sujetos participantes también coinciden en una serie de responsabilidades que le son propias al estudiante, de modo que se transforme en un proceso vinculante esencialmente a partir del reconocimiento del otro, (5). Entre estas responsabilidades declaradas destacan: mantener respeto hacia su tutor, disponerse positivamente al trabajo colaborativo con el, cumplir con las tareas que le son asignadas y ser criteriosos para solicitar ayuda de su tutor.

Finalmente, subyacen a las anteriores funciones y responsabilidades, una serie de propuestas y expectativas entre quienes participan de este proceso tutorial, que coinciden plenamente con las funciones planteadas por los autores anteriormente señalados $(5,4,9,11,13)$, dentro de las cuales destacan: la labor tutorial se enmarque en un contexto mas amplio de intervención con el propósito de mejorar la calidad de la formación; que el tutor ayude y acompañe al estudiantado en lo académico, administrativo y temas personales; que la labor tutorial sea una posibilidad de mejorar las relaciones entre docentes y docentes estudiantes. 
POBLETE G.C. \& GAMBOA J.R. Funciones, responsabilidades, propuestas y expectativas de la labor tutorial: opiniones de los docentes y estudiantes de la Escuela de Educación Física de la Pontificia Universidad Católica de Valparaíso. Mot.Hum. 13(1): 13-19; 2012.

\section{REFERENCIAS BIBLIOGRÁFICAS}

1. Gallego, S \& Riart, J. La tutoría y la orientación en el siglo XXI: Nuevas propuestas. Barcelona: Ed. Octaedro. 2006.

2. González, R \& Romo, A. Detrás del acompañamiento: una nueva cultura docente. México: ANUIES. 2005.

3. Enrique, C. \& Herrera, L. Proyectos de Innovación en Tutorías en la Universidad de Granada: Análisis de los instrumentos empleados. En Revista de Curriculum y Formación de Profesores 2008: 12 (2); 2 - 18.

4. Rodríguez, S. Manual de tutoria universitaria: Recursos para la acción. Barcelona: Ed. Octaedro. 2008.

5. Rubio, L. \& Santos, M. Programa institucional de tutorías Universidad pedagógica nacional unidad ajusco. Un proyecto en construcción. México: Universidad Pedagógica Nacional. 2004.

6. Gutiérrez, R. Evaluación curricular en el nivel medio superior de la universidad autónoma del estado de México: propuesta metodológico. En Espacios públicos 2009: 12 (24); 212-229.

7. Satulovsky, S. \& Theuler, S. Tutorías, un modelo para armar y desarmar: La tutoría en los primeros años de la escuela secundaria. Buenos Aires: Centro de Publicaciones Educativas y Material Didactico. 2009.

8. Hernández, M. Ruiz, M. \& Valencia, R. La tutoría académica: una estrategia para la adquisición de valores. En Revista de investigación educative. Universidad de veracruzana. México. 1999.
9. Sanz, R. Tutoría y atención personal al estudiante en la universidad: Madrid: Síntesis. 2009.

10. García, J. Ramos, D. \& Olivares, K. La función del tutor en ambientes presenciales y no presenciales. En Revista Mexicana de Orientación Educativa 2009: 6 (16); 16 - 19.

11. Lazo, C. \& Gabelas, J. La cercanía en la virtualidad: las tutorías en otra dimensión. En Quaderns Digitals. Revista de Nueva Tecnología y Sociedad 2008: 51.

12. Gómez, M. La tutoría: una nueva cultura docente. México: Universidad Autónoma de México. 2003.

13. Caepio, A. \& Guerra, L. Una experiencia de asesoramiento colaborativo como estrategia de apoyo a los profesores para la implementación de un programa de tutorías universitarias. En Profesorado. Revista de curriculum y formación de profesorado 2008: 1 - 12 .

14. Zabalza, M. El Prácticum en la Formación Universitaria: estado de la cuestión. En Revista de Educación 2011: (354): 21 - 46.

15. Carassai, M. Tutorías universitarias: un desafío actual. Argentina: Universidad Nacional de Quilmes. 2004.

16. Assael, J. Guzmán, I. \& Contreras, M. Qué entendemos por calidad de la educación. Santiago: Programa Interdisciplinario de Investigación en Educación PIIE. 1993.

17. Alvarez, J. El impacto del tutor en el nuevo modelo universitario cubano. Cuba: Instituto superior de ciencias médicas. 2010.

Dirigir Correspondencia a:

Carolina Poblete Gálvez

carolina.poblete@ucv.cl

Avda. El Bosque 1290, Santa Inés Viña del Mar

Chile

RECIBIDO: 25-05-2012

ACEPTADO: 25-06-2012 